\title{
Cancro do cólon e do recto: taxa de cobertura do rastreio e factores associados
}

Maria Espírito Santo, ${ }^{*}$ Elisa Ribeiro, ${ }^{* *}$ Tânia Colaço, ${ }^{* * *}$ Teresa Gomes****

\section{RESUMO}

Objectivos: Determinar a taxa de cobertura do rastreio de cancro do cólon e do recto (CCR), segundo o Plano Oncológico Nacional, e analisar factores socio-demográficos associados à sua realização.

Tipo de estudo: Observacional, analítico e transversal.

Local: Unidade de Saúde Familiar (USF) Horizonte e Centro de Saúde (CS) de Vila do Conde/Modivas.

População: Utentes inscritos nos USF Horizonte e CS de Vila do Conde/Modivas, com médico de família e idade compreendida entre 50 e 74 anos, no ano de 2006.

Métodos: Amostra aleatória de 504 indivíduos. Colheita de informação por processo documental, relativa a idade, sexo, escolaridade, número de doenças crónicas e realização do rastreio. Utilizaram-se os testes qui-quadrado, $t$ de Student e regressão logística binária (nível de significância de 0,05).

Resultados: Amostra com idade média de 60 anos e 57,3\% de mulheres. Obteve-se uma taxa de cobertura do rastreio de $24,6 \%$. Apenas foi encontrada relação estatisticamente significativa entre o número de doenças crónicas e a realização do rastreio, sendo que com um aumento do número de doenças crónicas se verifica mais frequentemente a realização do rastreio. $O$ aumento da idade também se relacionou com uma maior realização do rastreio; porém, quando ajustada para as outras variáveis, esta relação perdeu relevância estatística.

Conclusões: A taxa de cobertura encontrada é concordante com valores revelados em estudos de outros países. No entanto, o sexo, a idade e a escolaridade não se relacionaram com diferenças na realização do rastreio. Quanto ao número de doenças crónicas, dever-se-á ter em conta que talvez o seu aumento leve a um maior número de consultas o que pode originar mais oportunidades de rastreio. A baixa taxa de cobertura do rastreio de CCR deverá ser estudada para determinar outras variáveis passíveis de influenciar a sua realização.

Palavras-chave: Cancro do Cólon e do Recto; Rastreio.

\section{INTRODUÇÃO}

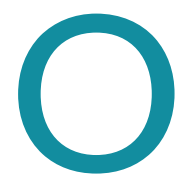

cancro do cólon e do recto (CCR) é o quarto tipo de cancro mais comum no mundo, sendo o mais frequente em determinadas regiões da Europa. ${ }^{1}$ Em Portugal não existem estatísticas nacionais actualizadas e fiáveis da incidência de doença tumoral maligna, ${ }^{2}$ no entanto, sabe-

\footnotetext{
*Médica de Família, Unidade de Saúde Familiar Porta do Sol, Unidade Local de Saúde de Matosinhos.

**Médica de Família, Unidade de Saúde Familiar Aqueduto, Agrupamento de Centros de Saúde Póvoa do Varzim / Vila do Conde (Grande Porto V).

***Médica de Família: (visitante), Co-op Community Health Centre, Victoria BC, Canadá.

****Médica de Família, Unidade de Saúde Familiar Corino de Andrade, Agrupamento de Centros de Saúde Póvoa do Varzim / Vila do Conde (Grande Porto V).
}

se que esta constitui a segunda causa de morte no país. ${ }^{3,4}$ Entre as doenças tumorais, o CCR representa actualmente a primeira causa de morte $^{3} \mathrm{e}$, contrariamente ao que se verifica na maioria dos países da Europa, a taxa de mortalidade por CCR em Portugal tem vindo progressivamente a aumentar. ${ }^{2,5,6}$ Como tal, este cancro constitui uma importante questão de saúde pública no nosso país. ${ }^{7}$

Existe evidência de que o rastreio de CCR permite uma detecção precoce de lesões e uma diminuição da mortalidade por CCR. ${ }^{8,9,10}$ A viabilidade deste rastreio advém do facto do CCR ser uma doença grave, com elevada prevalência e história natural longa e conhecida. 
Existe vantagem terapêutica com o diagnóstico precoce, estando provado que este pode reduzir a mortalidade por CCR em cerca de $20 \% .^{4,11,12}$ Além disso, estão disponíveis testes seguros, eficazes e acessíveis, com capacidade de rastreio e diagnóstico da doença numa fase inicial, obtendo-se uma boa relação custo-efectividade do rastreio. ${ }^{12,13}$

Em Portugal não existe um programa nacional de rastreio sistemático do CCR., ${ }^{2,45,12}$ No entanto, o Plano Oncológico Nacional (PON) apresenta as linhas orientadoras para que este seja implementado: ${ }^{7}$ «O cancro colo-rectal é, como se sabe, um importante problema de saúde pública. Dependendo da disponibilidade de profissionais treinados e das prioridades de recursos de cuidados de saúde, deverão ser organizados programas de rastreio por pesquisas de sangue oculto nas fezes e a colonoscopia de cinco em cinco anos nos indivíduos assintomáticos. O teste a utilizar será a pesquisa de sangue oculto nas fezes com convite ao grupo etário 50-74 anos e intervalo de rastreio de um-dois anos, sendo imediata a execução da colonoscopia em todos os casos com teste de sangue oculto positivo.»

O PON refere ainda que «Nos casos em que os programas de rastreio sistemático não sejam exequíveis deverão ser incentivados todos os meios conducentes à identificação de lesões pré-malignas ou de neoplasias iniciais sustentadas por critérios de qualidade.» ${ }^{7}$ Este plano foi integrado no Plano Nacional de Saúde (20042010) ${ }^{2}$ e no Plano Nacional de Prevenção e Controlo das Doenças Oncológicas 2007/2010, ${ }^{4}$ em vigor à data do estudo. Deste modo, o rastreio de CCR em Portugal é feito por detecção oportunista, cabendo aos médicos de família o convite (regra geral oportunista) para realização de PSOF a cada um ou dois anos.

Em Portugal, não existem dados sobre a taxa de cobertura do rastreio de CCR (quociente entre o número de indivíduos que realizaram o rastreio e o número total de indivíduos) mas, apesar disso, o Plano de Acção para a Saúde 2004 definia como meta para 2010 uma taxa de cobertura de $60 \% .{ }^{14}$ Vários estudos realizados nos Estados Unidos da América (EUA) obtiveram taxas de adesão ao rastreio de CCR entre $30-50 \%$. Contudo, estas não se tratam de taxas reais de cobertura, uma vez que, ao não discriminarem o motivo pelo qual os testes foram realizados, incluem nos rastreados os indivíduos que realizarem o teste como diagnóstico. ${ }^{15,16,17}$
Quando essa distinção foi considerada, a taxa de adesão ao rastreio foi de $25,8 \% .^{18}$

Apesar da evidência científica de que o rastreio reduz a incidência e a mortalidade por CCR, este continua a ser subutilizado. Determinando a taxa de cobertura do rastreio de CCR poderemos conhecer o estado actual de realização do mesmo. O resultado obtido deverá posteriormente ser enquadrado na meta nacional definida para $2010(60 \%)$.

O objectivo deste estudo é determinar a taxa de cobertura do rastreio de cancro do cólon e do recto na população entre os 50 e os 74 anos, inscrita em duas unidades de saúde, no ano de 2006. Pretende-se ainda analisar quais os factores socio-demográficos associados à realização do rastreio de CCR.

Hipótese nula $\left(\mathrm{H}_{0}\right)$ : A realização do rastreio de CCR é independente da idade, do sexo, da escolaridade e do número de doenças crónicas do indivíduo.

\section{MÉTODOS}

Foi realizado um estudo observacional, analítico e transversal com recolha de dados durante os meses de Junho e Julho de 2007 na Unidade de Saúde Familiar (USF) Horizonte e no Centro de Saúde (CS) de Vila do Conde/Modivas (Sede e Extensão das Caxinas).

A população estudada corresponde aos utentes inscritos na USF Horizonte e no Centro de Saúde de Vila do Conde / Modivas, com médico de família e com idade compreendida entre 50 e 74 anos, no dia 1 de Janeiro de 2006. Excluíram-se todos os indivíduos: colectomizados; com neoplasias benignas ou malignas do cólon e recto; com doença inflamatória intestinal, diverticulite ou síndromes hereditárias de CCR (polipose adenomatosa familiar e CCR hereditário sem polipose); com história familiar de CCR (mais de dois familiares de primeiro grau com CCR ou um familiar de primeiro grau com CCR diagnosticado antes dos 45-50 anos); sujeitos a pesquisa de sangue oculto nas fezes (PSOF) ou colonoscopia com fim diagnóstico; acamados, com demência ou patologia em estado terminal.

Seleccionaram-se aleatoriamente, por amostragem simples, 504 indivíduos. Os casos correspondentes aos critérios de exclusão foram repostos pelo indivíduo seguinte na lista aleatorizada. A dimensão da amostra foi calculada para uma taxa de cobertura esperada de $30 \%$ (metade do valor definido como meta para o ano de 
2010 pelo Plano de Acção para a Saúde ${ }^{14}$ e aproximado do valor encontrado num estudo semelhante realizado nos EUA $\left.^{18}\right)$, com uma precisão de $4 \%$ e um nível de confiança de $95 \%$.

As variáveis em estudo foram as seguintes:

- Idade - em anos a 1 de Janeiro de 2006;

- Género - feminino ou masculino;

- Escolaridade - analfabeto, primária, secundária, média, profissional, superior ou não registado na Ficha Individual do Processo Clínico;

- Número de doenças crónicas - número de doenças com uma ou mais das seguintes características: permanente, que deixe incapacidade residual, causada por alteração patológica não reversível, que requeira treino especial de reabilitação ou que se espera que leve à necessidade de um longo período de supervisão, observação ou cuidado;

- Realização do rastreio - realização de PSOF entre 01/01/2005 e 31/12/2006 ou de colonoscopia entre $01 / 01 / 2002$ e $31 / 12 / 2006$.

À data do estudo (2006), as unidades de saúde onde este decorreu eram: um Regime Remuneratório Experimental (USF Horizonte); a sede e uma extensão de um centro de saúde convencional (Centro de Saúde de Vila do Conde / Modivas). Todas se localizavam em ambiente urbano (apresentando a extensão de Caxinas características mistas por ser uma zona piscatória embora no perímetro das cidades da Póvoa de Varzim e de Vila do Conde), com bom acesso à realização de exames complementares (quer de rastreio, quer para conclusão do estudo em caso de rastreio positivo) e à referenciação a cuidados secundários em caso de diagnóstico de CCR. Nenhuma das unidades era ainda avaliada por indicadores de desempenho relativos a rastreios.

A recolha de informação foi efectuada por processo documental (consulta dos processos clínicos), tendo os dados sido registados em Microsoft Excel $2003^{\circledR} \mathrm{e}$ analisados em SPSS 15. $0^{\circledR}$. Determinaram-se resultados referentes à estatística descritiva e à estatística inferencial. De forma a estimar a relação de dependência entre a taxa de cobertura do rastreio e as diferentes variáveis, foram aplicados o teste de qui-quadrado, no caso das variáveis discretas, e o teste $t$ de Student quando se tratou da comparação de médias (variáveis contínuas). Posteriormente foi utilizada a regressão logística binária para exclusão de factores de confundimento. O ní- vel de significância adoptado foi de 0,05.

\section{RESULTADOS}

A amostra era constituída por 504 utentes com uma média de idades de 59,75 anos, sendo $57,3 \%$ do sexo feminino. A maioria dos indivíduos tinha a escolaridade primária $(71,4 \%)$, porém, só se obtiveram dados sobre escolaridade em 287 casos. No que se refere ao número de doenças crónicas, metade dos indivíduos $(48,9 \%)$ apresentava entre uma a duas, $31,5 \%$ mais de duas e $18,7 \%$ nenhuma doença.

A taxa de cobertura do rastreio obtida foi de $24,6 \%$ [IC (95\%): 21,0 - 28,6].

$\mathrm{Na}$ análise bivariada (Quadro I) verificou-se que as mulheres realizaram mais o rastreio. Contudo, esta diferença não apresentou significância estatística. Relativamente à escolaridade, as diferenças encontradas também não foram estatisticamente significativas, mas é importante realçar o facto de só haver disponibilidade de informação relativa a cerca de metade da amostra. No que se refere às doenças crónicas, verificou-se que a taxa de realização do rastreio foi significativamente superior nas pessoas com maior número de doenças crónicas. Em relação à idade (Quadro II) os in-

\begin{tabular}{|c|c|c|c|}
\hline \multicolumn{4}{|c|}{$\begin{array}{l}\text { QUADRO I. Análise bivariada (sexo, escolaridade, } \\
\text { número de doenças crónicas) }\end{array}$} \\
\hline & $\mathrm{n}$ total & Realizou (\%) & $p^{*}$ \\
\hline Sexo & & & 0,692 \\
\hline Feminino & 289 & 25,3 & \\
\hline Masculino & 215 & 23,7 & \\
\hline Escolaridade & & & 0,623 \\
\hline Analfabeto & 32 & 37,5 & \\
\hline Primária & 205 & 36,6 & \\
\hline Secundária & 28 & 25,0 & \\
\hline > Secundária & 22 & 40,9 & \\
\hline N. ${ }^{\circ}$ doenças crónicas & & & $<0,001$ \\
\hline Nenhuma & 94 & 4,3 & \\
\hline 1 ou 2 & 251 & 19,1 & \\
\hline$>2$ & 159 & 45,3 & \\
\hline
\end{tabular}

*teste do qui-quadrado; N. ${ }^{\circ}$ - número 


\begin{tabular}{|lccc|}
\hline \multicolumn{3}{|l|}{ QUADRO II. Análise bivariada (idade) } \\
& $\mathrm{n}$ total & Média $\pm D P$ & $P \dagger$ \\
\hline Idade & & & 0,001 \\
Realizou rastreio & 124 & $61,47 \pm 6,92$ & \\
Não realizou rastreio & 380 & $59,19 \pm 6,85$ & \\
& & & \\
\hline
\end{tabular}

tteste $t$ de Student

divíduos que realizaram o rastreio eram em média mais velhos do que os que não realizaram, e esta diferença foi estatisticamente significativa.

De forma a eliminar possíveis factores de confundimento, procedeu-se à análise multivariada por regressão logística binária (Quadro III). Constatou-se que apenas o número de doenças crónicas manteve influência estatisticamente significativa no cumprimento de rastreio. A idade, considerada em conjunto com as outras variáveis, perdeu significância estatística.

\section{DISCUSSÃO}

A taxa de cobertura do rastreio de CCR encontrada foi de $24,6 \%$, tratando-se de um valor baixo, muito aquém da meta definida para 2010 pelo Plano Nacional de Saúde (60\%). No entanto, este valor é concordante com os resultados apresentados em estudos de outros países. O estudo de Ata et al (EUA) revelou uma taxa de cobertura de $25,4 \%{ }^{18}$ enquanto que Sewitch et al, no Canadá, obtiveram o valor de $30,1 \% .{ }^{19}$ Os demais trabalhos encontrados revelaram taxas de cobertura superiores, mas não excluíram os casos nos quais os testes foram utilizados para diagnóstico ou realizados em indivíduos com risco aumentado de doença. ${ }^{15-17,20}$

Neste trabalho, a escolaridade e o sexo não foram associados à realização do rastreio, o que é discordante com outros trabalhos de investigação. Um maior nível de escolaridade tem sido relacionado com uma mais frequente realização do rastreio ${ }^{21,22,23} \mathrm{e}$, no que se refere ao sexo, diversos estudos encontraram diferenças significativas na realização do rastreio, não sendo consensuais quanto ao sexo que mais realiza o rastreio. ${ }^{15,21,22,24}$

O aumento da idade foi associado a uma maior realização do rastreio, o que é consistente com a literatura existente. ${ }^{15,19,21,22}$ Contudo, neste trabalho, quando

\begin{tabular}{|c|c|c|}
\hline \multicolumn{3}{|c|}{ QUADRO III. Análise multivariada } \\
\hline & $\begin{array}{l}\text { OR ajustado } \\
\text { (IC a } 95 \% \text { ) }\end{array}$ & $p^{*}$ \\
\hline Idade & $1,02(0,99-1,05)$ & 0,194 \\
\hline \multicolumn{3}{|l|}{ Sexo } \\
\hline Feminino & $0,94(0,60-1,45)$ & 0,765 \\
\hline Masculino & Classe referência & \\
\hline \multicolumn{3}{|c|}{ N. ${ }^{\circ}$ doenças crónicas } \\
\hline Nenhuma & Classe referência & \\
\hline 1 ou 2 & $5,25(1,83-15,02)$ & 0,002 \\
\hline$>2$ & $17,19(5,96-49,57)$ & $<0,001$ \\
\hline
\end{tabular}

* Modelo de regressão logística

efectuada a análise multivariada por regressão logística binária, a relação perdeu significância estatística. A idade não influenciou a taxa de cobertura do rastreio, sendo apenas uma variável de confundimento.

A taxa de realização do rastreio, neste estudo, foi significativamente superior nas pessoas com maior número de doenças crónicas. Esta relação também foi encontrada por Hawley et al, num estudo com metodologia sobreponível. ${ }^{16}$ Uma explicação possível é o facto de ter um maior número de doenças crónicas poder levar a um maior número de consultas e a mais oportunidades de rastreio.

Como ponto forte deste estudo salienta-se a selecção de uma amostra aleatória, que incluía indivíduos assintomáticos, sem qualquer história familiar. Além disso, a consulta de processos clínicos pode ser uma mais-valia quando comparada com dados recolhidos por auto-relato, por não estar sujeita a um viés de memória. Reforça ainda este trabalho o facto de a taxa obtida ser consistente com outros estudos.

Uma das limitações deste estudo é a não avaliação das causas que justifiquem a não realização do rastreio, nomeadamente o facto de ele ter sido, ou não, proposto a todos os pacientes que compunham a população e as circunstâncias de acessibilidade à realização dos exames. Ainda como limitações, é necessário ter em conta que os registos clínicos condicionaram a colheita de dados pela sua legibilidade e omissões. Acrescenta-se ainda o facto de este estudo considerar apenas o 
registo dos médicos de família, excluindo exames que possam ter sido realizados noutros contextos. Por fim, a indisponibilidade de dados relativos às características da população em estudo limita a generalização dos resultados obtidos.

Perante a taxa de cobertura do rastreio encontrada neste estudo, muito inferior à meta estabelecida no Plano Nacional de Saúde, será importante fazer estudos que permitam determinar a contribuição relativa de dois factores principais: a omissão do convite oportunista para o teste de rastreio pelo médico de família versus a não adesão dos indivíduos a este convite. Deste modo, será possível o planeamento de estratégias para adequar taxas de cobertura reais e metas.

\section{REFERÊNCIAS BIBLIOGRÁFICAS}

1. Parkin DM, Whelan SL, Ferlay J, Storm H; International Agency for Research on Cancer; International Association of Cancer Registries. Cancer incidence in five continents. IARC CancerBase. Lyon, 2005; 7. Disponível em: http://www.iacr.com.fr/statist.htm [acedido em 28/12/2010].

2. Portugal. Ministério da Saúde. Direcção-Geral da Saúde. Plano nacional de saúde 2004-2010: mais saúde para todos. Lisboa: Direcção-Geral da Saúde; 2004. Disponível em: http://www.dgsaude.min-saude.pt/ pns/capa.html [acedido em 28/12/2010].

3. Instituto Nacional de Estatística. Estatísticas da Saúde. Lisboa: INE; 2005. Disponível em: http://www.ine.pt [acedido em 28/12/2010].

4. Ministério da Saúde. Alto Comissariado da Saúde. Coordenação Nacional para as Doenças Oncológicas. Plano nacional de prevenção e controlo das doenças oncológicas 2007/2010 (PNPCDO). 2007. Disponível em: http://www.acs.min-saude.pt/pt/doencas-oncologicas [acedido em 28/12/2010].

5. Romãozinho JM. Editorial. Endonews. 2006; 16: 4-5.

6. Crabb C. European cancer programme reduced mortality, but mostly for men. Bulletin of the World Health Organization. 2003; 81 (9): 695. Disponível em: http://www.who.int/bulletin/volumes/81/9/News.pdf [acedido em 28/12/2010].

7. Plano Oncológico Nacional 2001-2005. Diário da República - Ia Série$B, n^{\circ} 190,17 / 8 / 2001,5241-7$.

8. Lindholm E, Brevinge $H$, Haglind $E$. Survival benefit in a randomized clinical trial of faecal occult blood screening for colorectal cancer. $\mathrm{Br} J$ Surg 2008 Aug; 95 (8): 1029-36.

9. Faivre J, Dancourt V, Lejeune C, Tazi MA, Lamour J, Gerard D, et al. Reduction in colorectal cancer mortality by faecal occult blood screening in a French controlled study. Gastroenterology 2004 Jun; 126 (7): 167480.

10. Hewitson P, Glasziou P, Watson E, Towler B, Irwig L. Cochrane systematic review of colorectal cancer screening using the faecal occult blood test (hemoccult): an update. Am J Gastroenterol 2008 Jun; 103 (6): 1541-9.
11. Asano TK, Toma D, Stern HS, McLeod RS. Current awareness in Canada of clinical practice guidelines for colorectal cancer screening. Can J Surg 2004 Apr;47(2):104-8.

12. Melo M, Braga R. Rastreio do cancro do cólon e do recto. Rev Port Clin Geral 2003 Set-Out; 19 (5): 471-82.

13. McLoughlin RM, O'Morain CA. Colorectal cancer screening. World J Gastroenterol 2006 Nov 14; 12 (42): 6747-50.

14. Portugal. Ministério da Saúde. Plano de acção para a saúde 2004 -áreas prioritárias. Lisboa; 2004. Disponível em: http://www.dgs.pt/upload/ membro.id/ficheiros/i006224.pdf [acedido em 28/12/2010].

15. Yeazel MW, Church TR, Jones RM, Kochevar LK, Watt GD, Cordes JE, et al. Colorectal cancer screening adherence in a general population. Cancer Epidemiol Biomarkers Prev 2004 Apr; 13 (4): 654-7.

16. Hawley ST, Vernon SW, Levin B, Vallejo B. Prevalence of colorectal cancer screening in a large medical organization. Cancer Epidemiol Biomarkers Prev 2004 Feb; 13 (2): 314-9.

17. Smith RA, Standiford CJ, Saraiya M. Actualizações sobre o rastreio do cancro do cólon, do colo do útero, da mama e da próstata. Patient Care (ed. Port.) 2006; 11 (111): 20-38.

18. Ata A, Elzey JD, Insaf TZ, Grau AM, Stain SC, Ahmed NU. Colorectal cancer prevention: adherence patterns and correlates of tests done for screening purposes within United States populations. Cancer Detect Prev 2006; 30 (2): 134-43.

19. Sewitch MJ, Fournier C, Ciampi A, Dyachenko A. Colorectal cancer screening in Canada: results of national survey. Chronic Dis Can 2008; 29 (1): 9-21.

20. Courtier R, Casamitjana M, Macià F, Panadés A, Castells X, Gil MJ, et al. Resultados de un estudio de cribado poblacional de neoplasia colorrectal. Cir Esp 2009 Mar; 85 (3): 152-7.

21. Zarychanski R, Chen Y, Bernstein CN, Hébert PC. Frequency of colorectal cancer screening and the impact of family physicians on screening behavior. CMAJ 2007 Sep 11; 177 (6): 593-7.

22. Chao A, Connell CJ, Cokkinides V, Jacobs EJ, Calle EE, Thun MJ. Underuse of screening sigmoidoscopy and colonoscopy in a large cohort of US adults. Am J Public Health 2004 Oct; 94 (10): 1775-81.

23. Liang SY, Phillips KA, Nagamine M, Ladabaum U, Haas JS. Rates and predictors of colorectal cancer screening. Prev Chronic Dis 2006 Oct; 3 (4): 1-13.

24. Meissner HI, Breen N, Klabunde CN, Vernon SW. Patterns of colorectal cancer screening uptake among men and women in the United States. Cancer Epidemiol Biomarkers Prev 2006 Feb; 15 (2): 389-94.

\section{CONFLITOS DE INTERESSE}

As autoras declaram não possuir qualquer tipo de conflitos de interesse.

\section{ENDEREÇO PARA CORRESPONDÊNCIA}

Maria Espírito Santo

Praceta de Recarei de Cima, 56

4465-339 Leça do Balio, Matosinhos

E-mail: maria_espiritosanto@yahoo.com

Recebido em 19/05/2009

Aceite para publicação em 03/05/2011 


\section{ABSTRACT}

\section{COLORECTAL CANCER SCREENING RATES AND ASSOCIATED FACTORS}

Aims: To determine the rate of colorectal cancer (CRC) screening in general practice and to analyse social and demographic factors associated with screening.

Study type: Descriptive.

Setting: Unidade de Saúde Familiar (USF) Horizonte and Centro de Saúde (CS) de Vila do Conde/Modivas.

Population: Patients aged 50 to 74 years old in 2006, registered with a family physician in USF Horizonte and CS de Vila do Conde/Modivas

Methods: A random sample of 504 people was selected. Age, sex, educational level, number of chronic diseases and data on completion of screening tests were collected from patient records. Chi-square test, t-Student test and logistic regression analysis were performed $(p=0,05)$.

Results: The sample had a mean age of 60 years and $57.3 \%$ of the patients were women. The screening rate for CRC was $24.6 \%$. There was a statistically significant association found between the number of chronic diseases and performance of screening. Age was associated with screening. However, when adjusted for other variables, this relationship was not found to be significant.

Conclusions: The screening rate for CRC was similar to that found in other international studies. Gender, age and educational level were not associated with screening. The presence of chronic diseases may lead to more medical consultations, which can provide more opportunities for screening. The low rate of screening found requires further study to determine other variables that may affect the performance of screening.

Keywords: Colorectal Cancer; Screening. 